\title{
PRESSURE BROADENING OF UV LINES
}

\author{
H. VAN REGEMORTER \\ Observatoire de Paris, 92 Meudon, France
}

\section{Introduction}

Most of the lines in the UV spectra are lines of ions which are formed in high temperature regions where the pressure broadening is caused by electrons and protons. This is the case in $\mathrm{O}$ and $\mathrm{B}$ type stars for which the theoretical calculation of the width of all the strong UV lines is important in determining both the blanketing effect and the abundances of the elements.

The cores of these strong lines are formed in non-LTE layers near the surface where the electron density is very low. The wings of some of the lines are more easy to interpret, being formed in deeper layers of the star, where one can assume LTE and where the electron density - or in the Sun, the neutral hydrogen density - is such that the pressure broadening is much more important than the natural width.

Two opposite approximations have been applied to the line broadening problem; the impact approximation is generally valid for electrons when the perturbations are so rapid that the collision time $\tau_{c}$ is very small compared to the typical time, $\Delta \omega^{-1}$, of importance in computing the profile at the frequency $\Delta \omega=\omega-\omega_{0}$ measured from the line centre. On the contrary, when $\tau_{c} \gg \Delta \omega^{-1}$ the quasi static approximation may be assumed. Both of these approximations have been considerably improved and efforts have been made recently to develop a unified theory valid from the impact regime to the static regime.

\section{The Impact Approximation for Electrons in the Case of Non-Hydrogenic Lines}

After the success of the Lindholm and Foley adiabatic theory, since the work of Baranger (1962), considerable progress has been made in calculating the electron broadening of non-hydrogenic lines for which the impact approximation is valid. Many review articles treat the subject in some detail (Traving, 1965; Van Regemorter, 1965; Wiese, 1965).

Until recently all calculations have been done using a semi-classical approximation, the perturber being considered as a classical particle, which is a good approximation when close collisions are not too important. This is the case at high energies when $k T$ is such that many inelastic transitions can take place between interacting levels. This is not the case when most of the collisions are adiabatic. Like the Lindholm theory the actual semi-classical theories fail to calculate these close collisions properly and, with the use of the particle flux conservation condition, are able to give only an estimate of this term, which is analogous to the old Weisskopf expression. But a comparison between experiments and semi-classical calculations, on one hand, and 
between semi-classical calculations and two recent quantum calculations, on the other, shows that the electron broadening of an atomic line can be represented using the semi-classical approximation. Accurate measurements and semi-classical calculations have been made for the resonance lines of $\mathrm{Mg}^{+}$and $\mathrm{Ca}^{+}$by Chapelle and Sahal-Brechot (1970) as well as quantum calculations by Bely and Griem (1970) and Barnes and Peach (1970). Until recently, the agreement between semi-classical calculations and numerous measurements was good only for neutral atoms for which one can use the data compiled in Griem's book (1964). For positive ions, theoretical calculations, using a straight classical path (Griem, 1964) have yielded Stark widths which are too low by factors between 2 and 10. Since the work of Brechot and Van Regemorter (1964), Van Regemorter (1965) and Sahal-Brechot (1969), all calculations have been done with hyperbolic paths for positive ions, the Coulomb attraction increasing the cross-sections at low energies. This improvement is also implicit in the semi-empirical approximation of Griem (1968), using an effective Gaunt factor and allowing for elastic collisions. Many differences still exist between the different semiclassical and empirical approximations which have been discussed in many recent papers (Roberts and Davis, 1968; Roberts, 1970; Sahal-Brechot, 1969; Griem, 1969). For positive ions, even at very low temperatures, the broadening, like the collision cross-sections, involves many values of the angular momentum $l$ of the perturbers, and, as is well known, the semi-classical approach becomes valid when $l$ is bigger than unity. On the other hand the broadening is given by a sum of averaged cross-sections $\sum_{j} N_{e} v Q_{i j}$ for which the particle flux conservation condition gives a good upper estimate of the close collisions. Many calculations and measurements have been done but only a few applications have been made to UV lines, these are for $\mathrm{Mg}^{+}$(Bely and Griem, 1970; Chapelle and Sahal-Brechot, 1970), $\mathrm{N}^{+}$and $\mathrm{C}^{+}$(Fortna et al., 1970).

It is important to note that the results are much better for simple atoms, for which all the atomic levels are known and for which accurate wave functions and oscillator strengths are available, than for complex atoms. For simple atoms the accuracy is better than $30 \%$. For complex atoms the accuracy is not so good, since other uncertainties are added to the uncertainties of the broadening problem itself.

For these non-hydrogenic lines the contribution of the protons to the broadening is of the order of $10 \%$ of the electron contribution. This contribution is calculated using an adiabatic approximation which is equivalent to the quasi static approximation at low temperature and converges to the impact approximation at high temperature and low densities (Griem et al., 1962).

\section{Improvements for the Calculation of Hydrogenic Lines}

Hydrogenic lines are those which are subject to the linear Stark effects, i.e. lines of hydrogenic ions, lines involving very excited states for which distance of two interacting levels, $a, a^{\prime}$ of different orbital quantum number, is not large compared to the width of the line $a, b$. Here it is necessary to apply the 'overlapping line' version of the impact approximation. For these lines, the application of the validity criterium $\tau$ 
compared with $\Delta \omega^{-1}$, to protons and electrons, gives the result that the protons are quasi-static in a large part of the profile and that the electrons have to be treated with the impact approximation in the line core and with the quasi-static approximation in the far wings.

First, the Holtsmark distributions for the ion field have to be corrected for ion-ion correlations and Debye shielding. These corrections have been calculated with a high accuracy by Hooper (1968a, b). Secondly, as is explained in all recent review articles, the impact approximation for electrons has to be modified in the case of overlapping lines, since all collisions inducing transitions between the Stark quasi-degenerate sublevels are not completed during the time $\Delta \omega^{-1}$. This means, in fact, a partial breakdown of the impact approximation; for some perturbers the collision time is much larger than $\Delta \omega^{-1}$. Until recently, this modification has been taken into account using the Lewis (1961) cutoff instead of the Debye cutoff for large impact parameters.

Earlier results for Balmer lines have been recalculated with this and many other improvements (lower state interaction, Stark splitting of the levels, accurate field distribution), for example by Kepple and Griem (1968), and good agreement with experiments (better than 10\%) has been found over a large domain of electron density and temperature. For these lines good agreement is found also for the line wings, using the latest interpolation formula by Griem (1968) between the impact regime and the quasi-static regime.

For Lyman- $\alpha$ the agreement between theory and experiment is not so good; the theoretical wings are much more 'quasi-static' $\left(\Delta \omega^{-2}\right.$ shape) than are the experimental ones. This discrepancy is not entirely removed with a more recent theory in which one of the approximations made in the usual impact approximation is removed.

In fact, in the usual impact approximation there are two main approximations:

(a) a binary encounter approximation: the strong collisions do not overlap in time; the collision time is much shorter than the time between two collisions;

(b) the complete collision approximation, which is valid when the collision time is much shorter than $\Delta \omega^{-1}$.

The second approximation has been removed (Smith et al., 1969a, b; Voslamber, 1969; Van Regemorter, 1969) in order to obtain a unified treatment for the electron contribution which is valid from the usual impact regime to the quasi static regime of the line wings. The more elaborated treatment has been given by Smith et al. (1969b) and has been applied to the profile of Lyman- $\alpha$. The residual disagreement may be explained by the approximate treatment of strong collisions using a classical path and by the failure of approximation a. An interesting attempt has been made recently to remove the latter by using an exact solution of a simple model of the microfield, its probability distribution and the covariance of its fluctuations (Brissaud and Frisch, 1970).

On the other hand much work remains to be done for transitions involving high quantum number $n^{\prime}$ for which - as has been noted before - the line wings are much more quasistatic than predicted by the usual theory (Schluter and Avila, 1966; Pfennig, 1966). 


\section{Pressure Broadening Due to Neutral Hydrogen}

This kind of broadening is not important for most of the lines in the UV spectrum and will be examined briefly. In fact, some line wings of the solar UV spectrum are formed where the density of neutral $\mathrm{H}$ is much higher than the electron density. It is still currently assumed that the broadening by $\mathrm{H}$ is given by the long range Van der Waals interaction, $C R^{-6}$. There is now much experimental and theoretical evidence that the broadening due to collisions with light atoms like $\mathbf{H}$ or $\mathrm{He}$ does not arise from the Van der Waals interaction but from a shorter range interaction. Even at low thermal temperatures, the broadening - such as the relaxation of excited states due to the transfer of excitation between fine structure levels - cannot be explained by the Van der Waals theory. In the case of collisions with neutral helium, the polarizability and weight of which is comparable to hydrogen, this is confirmed by many experiments (Behmenburg, 1964; Hindmarsh et al., 1967; Roueff, 1970) and by theory (Roueff, 1970).

Calculations are in progress for hydrogen (Roueff and Van Regemorter, 1969) for which no experiment is yet available; the broadening is not given by the Van der Waals interaction, and moreover, if this is true at thermal temperatures, it is true, a fortiori, at the temperature of the Sun.

On the other hand, the usual impact approximation is currently assumed (Lindholm formula) for the broadening due to neutral atoms, but this is not always valid. In this case, as in the case of charged particles, one can remove approximation $b$ (see above) and use adiabatic theory, valid for the entire profile (Hindmarsh and Farr, 1969; Takeo, 1970).

\section{References}

Baranger, M.: 1962, in Atomic and Molecular Processes (ed. by D. R. Bates), Academic Press.

Barnes, K. and Peach, G.: 1970, J. Phys. B. [2], 3, 350.

Behmenburg, W.: 1964, J. Quant. Rad. Spectr. Trans. 4, 177.

Bely, O. and Griem, H. R.: 1970, Phys. Rev. 1A, 97.

Brechot, S. and Van Regemorter, H.: 1964, Ann. Astrophys. 27, 432.

Brissaud, A. and Frisch, U.: 1970, to be published in J. Quant. Rad. Spectr. Trans.

Chapelle, J. and Sahal-Brechot, S.: 1970, Astron. Astrophys. 6, 415.

Fortna, J. E., Elton, R. C., and Griem, H. R.: 1970, to be published in Phys. Rev.

Griem, H. R.: 1964, Plasma Spectroscopy, McGraw-Hill Publ. Co.

Griem, H. R.: 1966, Phys. Rev. Letters 17, 509.

Griem, H. R.: 1968, Phys. Rev. 165, 258.

Griem, H. R.: 1969, Comments Atomic Mol. Phys. 1, 27.

Griem, H. R., Baranger, M., Kolb, A. C., and Oertel, G.: 1962, Phys. Rev.125, 177.

Hindmarsh, W. R. and Farr, J. M.: 1969, J. Phys. B. [2], 2, 1388.

Hindmarsh, W. R., Petford, A. D., and Smith, G.: 1967, Proc. Roy. Soc. A207, 296.

Hooper, C. F.: 1968a, Phys. Rev. 165, 215.

Hooper, C. F.: 1968b, Phys. Rev. 169, 193.

Kepple, P. and Griem, H. R.: 1968, Phys. Rev. 170, 317.

Lewis, M.: 1961, Phys. Rev. 121, 501.

Pfennig, H.: 1966, Z. Naturforsch 21a, 1648.

Roberts, D. E.: 1970, Astron. Astrophys. 6, 1.

Roberts, D. E. and Davis, J.: 1968, J. Phys. B. [2], 1, 48. 
Roueff, E.: 1970, Astron. Astrophys. 7, 4.

Roueff, E. and Van Regemorter, H.: 1969, Astron. Astrophys. 1, 69.

Sahal-Brechot, S.: 1969a, Astron. Astrophys. 1, 91.

Sahal-Brechot, S.: 1969b, Astron. Astrophys. 2, 322.

Schluter, H. and Avila, C.: 1966, Astrophys. J. 144, 785.

Smith, E. W., Cooper, J., and Vidal, C. R.: 1969a, Nat. Bur. Std. J. Res. 73A, 389.

Smith, E. W., Cooper, J., and Vidal, C. R.: 1969b, Phys. Rev. 185, 140.

Takeo, M.: 1970, Phys. Rev. 1a, 1143.

Traving, G.: 1965, in Plasma Diagnostics (ed. by Lochte-Holtgreven), North-Holland Publ. Co., Amsterdam.

Van Regemorter, H.: 1965, Ann. Rev. Astron. Astrophys. 3, 71.

Van Regemorter, H.: 1969, Phys. Letters, 30A, 365.

Voslamber, D.: 1969, Z. Naturforsch, 24a, 1458.

Wiese, W. L.: 1965, in Plasma Diagnostic Techniques, Academic Press.

\section{DISCUSSION}

D. D. Burgess: I would like to caution that, for the type of resonance lines discussed by Dr. Morton this morning - i.e. specifically the resonance lines of Li-like ions - there may be problems not only with the line-widths, but also with the profiles. All existing line-shape calculations would take the profile as Lorentzian for these lines. This is because such theories neglect the coupling between the perturbing (free) electrons and the transverse radiation field. For many lines this effect is negligible. However for Li-like resonance lines of high ionization stages this interaction can significantly distort the profile, increasing the opacity in the blue wing, and sharply decreasing it below the value expected for a Lorentzian in the red wing, thus possibly altering line-blanketing effects etc.

$L$. H. Aller: Can you estimate the factor by which we should multiply the Van der Waals damping constants for FeI lines broadened by collisions with hydrogen atoms? Empirical factors of three to five are suggested by Ross' work.

$H$. Van Regemorter: Our work on the broadening by $\mathrm{H}$ is not completed yet. For the case of the broadening of alkali atoms by $\mathrm{He}$, results are given in a recent issue of Astron. Astrophys. and are compared to different experiments by Hindmarsh and Rostas. The Van der Waals contribution is so small, even at thermal temperature, that it can be neglected.

D. D. Burgess: You mentioned the Unified Theories of Cooper, Vidal and Smith, and of Voslamber, and showed comparisons with two experiments on Ly- $\alpha$. I would comment that not only do we clearly need a third experiment, but that it seems to me that the validity of these theories in the intermediate regime is not yet fully established mathematically. Clearly they are asymptotic to both impact and quasi-static (nearest neighbour) regimes. In the intermediate regime their validity depends on being able to interchange the order of a summation and an integration, and I personally feel that the convergence properties need more examination at this stage. 